\title{
Dynamic Memory Efficient Frequent Pattern Growth for Data Excavation
}

\author{
G. Gunasekaran \\ Assistant Professor of Computer Science \\ Nehru Memorial College \\ Puthnampatti, Trichy
}

\author{
S. Murugan, $\mathrm{PhD}$ \\ Associate Professor of Computer Science \\ Nehru Memorial College \\ Puthnampatti, Trichy
}

\begin{abstract}
Advancements in information technology increase the data volume of many domains into manyfold. Dynamic Memory Efficient Frequent Pattern (DMEFP) technique introduces new methods to represent data and redundant frequent patterns. Introduction of Repeat Pattern Table (RPT) and new node type 'Tree Pattern Node' (TPN) in frequent pattern tree softens the data mining process to be performed in a modern way. DMEFP technique comprises new rules to aggregate pattern nodes and RPT. Computational resources are used sagely in DMEFP technique for data mining. Reduced resource consumption helps to parse large amount of data in short time durations without much complexity.
\end{abstract}

\section{Keywords}

Information Technology, Data Mining, FP-Growth, Frequent Pattern Tree, Memory efficient data mining

\section{INTRODUCTION}

Finding correlations between associated data create a valuable cognition. Modern databases are huge in volume and require complex procedures for parsing. Data mining techniques are designed to perform deviation detection, dependency modelling (association rule learning), clustering, classifying, regression and summarization. Association rules are used to relate data items in a procedural way. Deviation detection otherwise called as Anomaly detection is a process of identifying unusual records in a dataset. An anonymous record requires special investigation to find whether the transaction is occurred by mistake or a special rule has to be introduced to validate the record. Dependency modelling is used to define the relationships between the variables in the transaction records. Clustering is used to find and organize same or similar group of records under a common label. Classification procedure is used to identify a new record based on the existing known structures created by clustering. Regression is used to introduce a common function to correlate the data of same group with no or least errors. This is used to estimate the relationships within records or datasets. Summarization is used to enable data structure visualizations and to produce reports based on different requirements.

\section{EXISTING SYSTEMS}

\subsection{Apriori}

Apriori[1] is a standard well known procedure for extracting association rules from binary transaction databases. Apriori starts with a frequent individual item in a dataset and broadens it to the frequent item sets in a dataset. In Apriori algorithm, the frequency of the item sets are handled using a threshold value of $\boldsymbol{C}$. Apriori uses 'Candidate Generation' a bottom-up approach to extend a single frequent item into frequent item sets. This process continues until there are no successful Candidate Generations. Candidate Item sets' count is calculated using Hash-Tree structure and breath-first-search by Apriori. It can generate candidate item set with length of $\boldsymbol{k}$ from item set length $\boldsymbol{k}-\mathbf{1}$. Then the frequent item sets are determined from the candidates.

If $\mathrm{T}$ is a transaction database with support threshold $\in$ then the pseudo code for Apriori will be

$\operatorname{Apriori}(T, \epsilon)$

$\mathrm{L} 1=\{$ Large $1-$ itemsets $\}$

$\mathrm{K}=2$

while $L_{k-1} \neq \emptyset$

$C_{k}=\left\{a U\{b\} \mid a \epsilon L_{k-1} \wedge b \notin a\right\}-\{c \mid\{s \mid s \subseteq c \wedge$

$\left.|\mathrm{s}|=k-1\} \nsubseteq L_{k-1}\right\}$

for transactions $t \in T$

$$
C_{t}=\left\{c \mid c \in C_{k} \wedge c \subseteq t\right\}
$$

for candidates $c \in C_{t}$

$$
\operatorname{count}[c]=\operatorname{count}[c]+1
$$

$L_{k}=\left\{\mathrm{c} \mid \mathrm{c} \in C_{k} \wedge \operatorname{count}[c] \geq \in\right\}$

$k=k+1$

return $\bigcup_{k} L_{k}$

where $C_{k}$ is the candidate set for level $k$.

In Apriori algorithm, Candidate Generation is a resource consuming process that involves large number of subsets scans. Bottom-up approach in breadth-first traversal can find maximum subset count only after finding all $2^{|s|}-1$ proper subsets. This disadvantage makes it difficult to use Apriori algorithm with larger database. To overcome this issue some other procedures are introduced.

\subsection{FP-Growth}

FP-Growth[2] technique is based on FP-Tree construction first phase process helps to extract frequent item sets which is the second phase process. Here frequent item sets are extracted directly form FP-Tree therefore resource consuming candidate generation process is eliminated. FP-Tree is constructed by scanning database to find support count for each item. Rare items are eliminated. Frequent item sets are arranged in descending order based on their support count. Nodes are used to represent the items and facilitated with a counter. FP-Growth reads a transaction and maps it to a path. Counters are incremented whenever there is a overlap found the particular path. Single linked list is used to maintain the FP-Tree[3][4]. When all transactions share a same path, then there will be a single path in the FP-Tree and this is considered as the best scenario for FP-Growth. If all transactions are performed with unique items - that is there are 
no items in common between transactions, then the size of the FP-Tree will be as large as the actual data.

FP-Tree Structure:

1. Root Node (Null Node) which holds item-prefix sub-trees is used to initializes tree structure

2. Each item prefix sub-tree node consists three fields \{Item Name, Count, Next Node Link\}

3. Frequent-Item-header table consists of two fields \{Item Name, Link to first node \}

4. Frequent -Item-header table has optional support count for an item

FP-Tree Construction Pseudo code:

FPTree ConstructFPTree(Transaction Database DB)

1. Perform DB scan, Accumulate F (set of frequent items) and the support of each frequent item. Sort $F$ in support-descending order in the list of frequent items named FList

2. Initialize Tree with Root Node T (null Node)

3. For each transaction Trans in DB do the following:

i. Assort frequent items from transactions and sort them based on the order of FList.

ii. Represent sorted frequent-item list as $[p \mid P]$, where $p$ is the first element and $\mathrm{P}$ is the remaining list

iii. If $\mathrm{T}$ has a child $\mathrm{N}$

then item-name $=p$

$\mathrm{N}=\mathrm{N}+1$

Else Create new node $\mathrm{N}$ with count 1

iv. Repeat step iii recursively for full database

The size of the FP-Tree depends on the order of the items. Therefore the memory consumption of FP-Growth is very high in general when large databases are involved.

\subsection{ECLAT}

Eclat[5] is recursively defined procedure to perform item set mining that is used to frequent pattern identification. These frequent patterns are also called as association rules. Eclat uses Tidset[6] intersections to compute candidate item set support count and avoids generation of candidate subsets those are not present in prefix tree.

The initial Eclat call uses all the single item sets with their transaction IDs (tids). Then the recursive calls are initialized to verify all itemset-tidset pair $\langle X, t(X)\rangle$ with other pairs $\langle Y, t(Y)\rangle$ to generate new candidates $N_{X Y}$. Whenever the new candidates is found to be frequent, then it is added with set $P_{X}$.

Pseudo code:

1. Let $\mathrm{S}$ be an Item set

2. $\mathrm{T}$ is the transaction tag

3. Multiset transaction $\mathrm{U}=\{X \in T \mid S \subseteq t\}$

4. Absolute Support of $\mathrm{S}=|U|=\quad|\{X \in T \mid S \subseteq t\}|$
5. Relative support of $\mathrm{S}=(|U| /|T|) * 100 \%$; where $|U|$ and $|T|$ are the number of elements in $U$ and $T$ respectively

6. The anti-monotone support is $\forall I, J:(J \subseteq I)=(\operatorname{supp}(J) \geq$ $\operatorname{supp}(I))$

Eclat's Depth-First-Search procedure and its vertical database layout causes more time for intersection of Tidlists. When the database is considerably large, the memory consumption of Eclat's is large and occupying other computational resources makes it less manageable.

\subsection{DFEM}

DFEM[7] refers Dynamic FP-Tree and Eclat Method. It uses both FP-Growth and Eclat algorithms for mining. A threshold value is used to determine the switching process between FPGrowth and Eclat algorithm. DFEM has four major modules, they are Construction of FP-Tree, Mining FP-Tree, Mining Bit Vector and updating the threshold.

FP-Tree Construction Procedure:

1. Find frequent items by scanning the database

2. Construct FP-Tree based on the scan results

3. Call FP-Tree Mining

FP-Tree Mining Procedure:

1. If FP-Tree has a single path, then all combinations of $X$ nodes $\mathrm{P}=\mathrm{X} \cup$ suffix

2. For all Items of $\mathrm{Y}$ in the header table $\mathrm{P}=\mathrm{Y} \cup$ suffix

3. Construct Conditional pattern of $\mathrm{Y}$ based on $\mathrm{C}$

4. If number of nodes in $\mathrm{Y}>\mathrm{K}$, then construct $\mathrm{Y}$ 's conditional FP-Tree and call FP-Mining Procedure recursively

5. Else transform C into Bit Vector V, Weight Vector W and call Bit Vector Mining

Bit Vector Mining Procedure:

1. Arrange V based on its item support in descending order

2. For each vector $v_{i}$ in $\mathrm{V}$

$$
\text { Output }=v_{i} \cup \text { suffix }
$$

3. For each vector $v_{k}$ in $\mathrm{V}$, where $\mathrm{k}<\mathrm{i}$

$$
u_{k}=v_{i} \text { AND } v_{k}
$$

4. $\sup _{k}=$ support of $u_{k}$ based on weight $w$

5. If all $u_{k}$ in $\mathrm{U}$ are identical to $v_{i}$, then for each combination of $\mathrm{X}$ in $\mathrm{U}$ output $=\mathrm{X} \cup$ output

else if $U$ is empty, then call Bit Vector Mining again

Threshold updating procedure:

In DFEM, FP-Tree mining[8] has a set of values of threshold $\mathrm{K}$ as $\mathrm{K}=\left\{k_{0}, k_{1}, k_{n}\right\}$. The difference between previous pattern $P_{i-1}$ and current pattern $P_{i}$ is represented as $R_{i} . R_{i}$ is calculated as

$$
R_{i}=\frac{P_{i-1}}{P_{i},(i=1 \text { to } N)}
$$

The value of $\mathrm{K}$ is finalized using the condition $\left(\nexists R_{j}>2, \forall j>k\right) \in R_{i}<2$ 
Threshold updating pseudo code:

1. If Update $\mathrm{K}$ called for the first time

Then Create an array $\mathrm{P}$ with $\mathrm{N}$ elements.

2. Initialize the array with zero

3. For $\mathrm{i}=0$ to $\mathrm{N}-1$

$$
\text { If size }>\mathrm{I} * \text { size then } P_{i}=P_{i}+\text { New }
$$

Pattern

$$
\text { Else exit loop }
$$

4. Initalize $\mathrm{K}$ as Zero $(\mathrm{K}=0)$

5. For $\mathrm{i}=\mathrm{N}-1$ to 1

$$
\text { If } R_{i} \geq 2 \text { then } \mathrm{K}=(\mathrm{i}+1) * \text { step }
$$

6. exit loop

DFEM is quicker and more accurate than the previous methods. But the memory consumption is comparatively high when dealing with a larger size database.

\subsection{MEFP}

Memory Efficient Frequent Pattern mining[9][10] uses transposition of database. The space complexity of MEFP is $O(n)$ and the longest common sequence space complexity is $O\left(n^{2}\right)$. The time consumption of MEFP is $O(m n)$. It uses X data or Y data based on the quantity of support counts. The space is either $O(m)$ or $O(n)$ whichever is smaller.

MEFP Procedure:

1. Convert Database DB into Transpose Form DT

2. Compute F1 for all frequent Items

3. $\mathrm{C} 1=\mathrm{DT}$ [Frequent Item Row with Transaction ID String]

4. Assign $\mathrm{K}=2$

5. While Lk- $1 \neq\{K\}$ do

Compute $C_{k}$ for all candidates k-1 item sets

Compute $\mathrm{Lk}=\operatorname{APS}\left(C_{k}\right)$

Increment the value of $\mathrm{K}$ by 1

In MEFP, there is a need to shift rows and columns and X-Y variable values interchanging[11][12] takes more time. Even though MEFP consumes lesser memory, the time for formatting frequent patterns and generating corresponding association rules takes more time for larger databases.

\section{PROPOSED METHOD}

Dynamic Memory Efficient Frequent Pattern (DME-FP) procedure introduces a new node type named Tree Pattern Node (TPN). It also introduces Repeat Pattern Table (RPT) a table to manage TPN. This procedure is designed with caution to handle computational resources[13][ like memory[14] and time[15] with improved basic metrics of a data mining procedure of accuracy, precision and recall.

Table 1 contains a transaction history with different item sets of items $\{a, b, c, d, e, j, k, 1, m, n\}$. As given in table, the transactions can be any possible combination of the taken items.

Table 1

\begin{tabular}{||c|c|}
\hline TID & Items \\
\hline 1 & $\{\mathrm{a}, \mathrm{b}\}$ \\
\hline 2 & $\{\mathrm{~b}, \mathrm{c}, \mathrm{d}\}$ \\
\hline 3 & $\{\mathrm{a}, \mathrm{c}, \mathrm{d}, \mathrm{e}\}$ \\
\hline 4 & $\{\mathrm{a}, \mathrm{d}, \mathrm{e}\}$ \\
\hline 5 & $\{\mathrm{a}, \mathrm{b}, \mathrm{c}\}$ \\
\hline 6 & $\{\mathrm{a}, \mathrm{b}, \mathrm{c}, \mathrm{d}\}$ \\
\hline 7 & $\{\mathrm{a}\}$ \\
\hline 8 & $\{\mathrm{a}, \mathrm{b}, \mathrm{c}\}$ \\
\hline 9 & $\{\mathrm{a}, \mathrm{b}, \mathrm{d}\}$ \\
\hline 10 & $\{\mathrm{~b}, \mathrm{c}, \mathrm{e}\}$ \\
\hline 11 & $\{\mathrm{c}, \mathrm{j}, \mathrm{k}\}$ \\
\hline 12 & $\{\mathrm{c}, \mathrm{k}, \mathrm{l}, \mathrm{m}\}$ \\
\hline 13 & $\{\mathrm{c}, \mathrm{j}, \mathrm{l}, \mathrm{m}, \mathrm{n}\}$ \\
\hline 14 & $\{\mathrm{c}, \mathrm{j}, \mathrm{m}, \mathrm{n}\}$ \\
\hline 15 & $\{\mathrm{c}, \mathrm{j}, \mathrm{k}, \mathrm{l}\}$ \\
\hline 16 & $\{\mathrm{c}, \mathrm{j}, \mathrm{k}, \mathrm{l}, \mathrm{m}\}$ \\
\hline 17 & $\{\mathrm{c}, \mathrm{j}\}$ \\
\hline 18 & $\{\mathrm{c}, \mathrm{j}, \mathrm{k}, \mathrm{l}\}$ \\
\hline 19 & $\{\mathrm{c}, \mathrm{j}, \mathrm{k}, \mathrm{m}\}$ \\
\hline 20 & $\{\mathrm{c}, \mathrm{k}, \mathrm{l}, \mathrm{n}\}$ \\
\hline
\end{tabular}

A typical FP-Tree structure will be formed as in Figure 1 

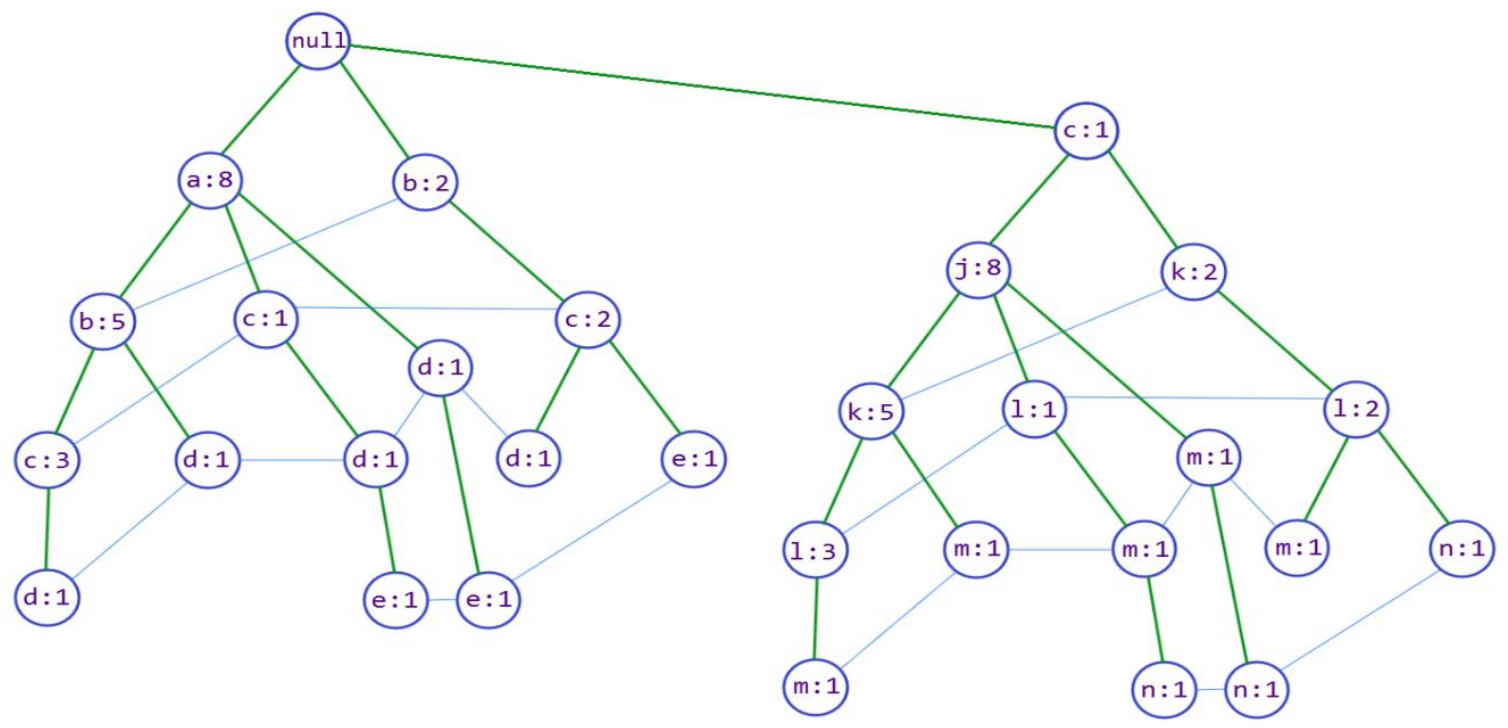

Figure 1

If the size of a node is declared as $\sigma$, then the size of the tree will be $30 \sigma$. A typical FP-Tree node with three child nodes consumes $768(\sigma)$ bits on average. Table 2 shows memory allocation of a typical FP-Tree Node

Table 2

\begin{tabular}{||l|c|}
\hline \hline Purpose & Size(bits) \\
\hline Parent Address & 64 \\
\hline Transaction Item-ID & 416 \\
\hline Count & 64 \\
\hline Child Node Count (nc) & 32 \\
\hline Child Node Address & $\mathrm{nc} * 64$ \\
\hline
\end{tabular}

Therefore to construct a FP-Tree as shown in Figure 2, the memory consumption will be 23040 Bytes. This implies to handle twenty transactions with ten different items FP-Tree method requires 2880 Bytes of memory. Modern databases contain vast number of items with large number of transactions. To organize a complete database into FP-Tree for analysis will be a memory starving process.

Proposed DME-FP uses a different type of tree nodes. Since DME-FP has an additional node type of TPN, one more field with single bit length is added with the default FP-Tree Node. So the size of a DME-FP node will be 769 bits. DME-FP node architecture is given in Table 3
Table 3

\begin{tabular}{||l||c||}
\hline Purpose & Size(bits) \\
\hline \hline Node Type & 1 \\
\hline \hline Parent Address & 64 \\
\hline \hline Transaction Item-ID & 416 \\
\hline \hline Count & 64 \\
\hline \hline Child Node Count (nc) & 32 \\
\hline \hline Child Node Address & $\mathrm{nc} * 64$ \\
\hline \hline
\end{tabular}

The Node Type field will have the value 0 is to represent a regular node and 1 is to represent a Tree Pattern Node. In DME-FP, a RPT consists of four fixed length fields and a variable length field. Pattern ID, Root Node ID, Number of nodes in BFS and Number of Substitute Items Count are fixed with 16 bits, 64 bits, 8 bits and 4 bits respectively. The Number of nodes in BFS filed can hold up to 255 refers a pattern can have

255 nodes maximum including the root node. The fifth variable length field is based on the fourth field Substitute Item Count. If the value of Substitute Item count is $\eta$ then the size of fifth filed will be $\eta \times 416$ bits. Since the size of Substitute Item Count is limited to 4 bits, the value of $\eta$ can go up to 16 . This refers the number of substitute items can be 16 at maximum.

For the transaction history as in Table 1, Repeat Pattern Table will be generated as follows

\begin{tabular}{|c|c|c|c|c|}
\hline $\begin{array}{c}\text { Pattern } \\
\text { ID }\end{array}$ & Root Node ID & $\begin{array}{c}\text { Number of Nodes } \\
\text { on BFS }\end{array}$ & $\begin{array}{c}\text { Substitute Item } \\
\text { Count }\end{array}$ & $\begin{array}{c}\text { Substitute } \\
\text { Items }\end{array}$ \\
\hline 1 & Address (null) & $15(00001111)$ & $5(0101)$ & $j, k, 1, m, n$ \\
\hline
\end{tabular}




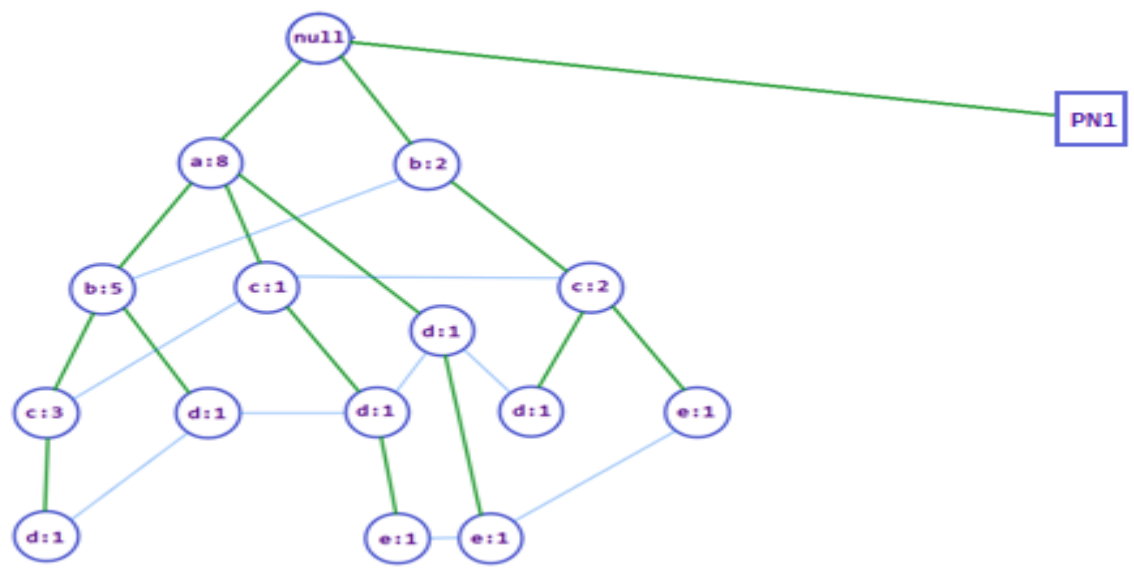

Figure 2

The size of this RPT is 1664 bits. The DME-FP tree representation for the transaction history is shown in Figure 2.

This DME-FP Tree the size of 15 regular nodes is 11535 bits, the size of 1 tree pattern node is 577 bits and the size of RPT is 1664 bits. In total, the DME-FP tree consumes 13776 bits (12112 bits for tree +1664 for RPT) whereas regular FP-Tree consumes 23040 bits for the same transaction history. Content of tree pattern node PN1 is given in table 4 .

Overall memory preservation of DME-FP over regular FPTree is 9264 bits (1158 Bytes). This proves using DME-FP saves greater than $1 \mathrm{~KB}$ of memory for a 20 item set transaction history.

Table 4

\begin{tabular}{||l|c|r||}
\hline \hline Field & Value & Size (Bits) \\
\hline Node Type & 1 & 1 \\
\hline Parent Address & Address(null) & 64 \\
\hline $\begin{array}{l}\text { Transaction } \\
\text { Item ID }\end{array}$ & 11 & 416 \\
\hline Count & 1 & 64 \\
\hline $\begin{array}{l}\text { Child Node } \\
\text { Count }\end{array}$ & 0 & 32 \\
\hline $\begin{array}{l}\text { Child Node } \\
\text { Address }\end{array}$ & 0 & 0 \\
\hline \multicolumn{2}{|c|}{ Total Size } & 577 \\
\hline
\end{tabular}

\section{EXPERIMENTAL SETUP}

To evaluate the performance of DME-FP procedure, benchmark datasets Chess, Kosarak, Mushroom, pumbsb_star, Retail and T10I4D100K are used. Accuracy, Precision, Recall, Processing Time and Memory are measured with noted datasets and for a comparative analysis, existing methods Apriori, FP-Growth, Eclat, DFEM and MEFP are taken. The processing time and memory consumption are measured in a computer with Processor $2.4 \mathrm{GHz}$ i5-4210U Quad-core processor and 4GB RAM. Visual Studio IDE is used to develop the user interface and $\mathrm{VC}++$ programming language is used to code the proposed method. Standard data mining libraries are used to evaluate existing methods. Readings are measured up to 100000 records in equal interval of 10000 records for all methods.

\section{RESULTS AND ANALYSIS}

Accuracy is one of the vital parameter in data mining. It refers number of correct predictions over total number of predictions. The higher accuracy indicates the higher stability of the data mining procedure. DME-FP achieved highest accuracy of $92.92 \%$ with the average of $91.25 \%$. The nearest accuracy is achieved by MEFP. MEFP gains $89.91 \%$ of highest accuracy with the average of $88.89 \%$. Remaining methods Apriori, FP-Growth, Eclat and DFEM are scoring $79.91 \%, 82.43 \%, 85.45 \%$ and $86.64 \%$ of accuracy respectively.

The accuracy report is given in table 5 and compared in Figure 3.

Table 5

\begin{tabular}{||c|c|c|c|c|c|c||}
\hline \hline \multicolumn{7}{|c|}{ Accuracy $(\%)$} \\
\hline Records & Apriori & FP-Growth & Eclat & DFEM & MEFP & DME-FP \\
\hline 10000 & 80.56 & 83.59 & 86.63 & 87.66 & 89.69 & 92.73 \\
\hline 20000 & 80.98 & 81.24 & 84.51 & 85.78 & 87.37 & 90.63 \\
\hline 30000 & 79.44 & 83.96 & 85.49 & 85.01 & 88.53 & 90.05 \\
\hline 40000 & 80.42 & 82.12 & 84.5 & 87.2 & 88.58 & 90.29 \\
\hline 50000 & 79.64 & 82.12 & 85.27 & 85.75 & 87.9 & 91.06 \\
\hline 60000 & 79.86 & 81.76 & 84.35 & 87.94 & 88.85 & 91.43 \\
\hline 70000 & 78.55 & 81.56 & 86.89 & 87.9 & 89.91 & 92.92 \\
\hline 80000 & 79.2 & 83.96 & 85.72 & 85.47 & 89.23 & 90.98 \\
\hline 90000 & 80.91 & 81.61 & 85.32 & 87.03 & 89.73 & 90.44 \\
\hline 100000 & 79.59 & 82.39 & 85.87 & 86.66 & 89.14 & 91.94 \\
\hline \hline
\end{tabular}




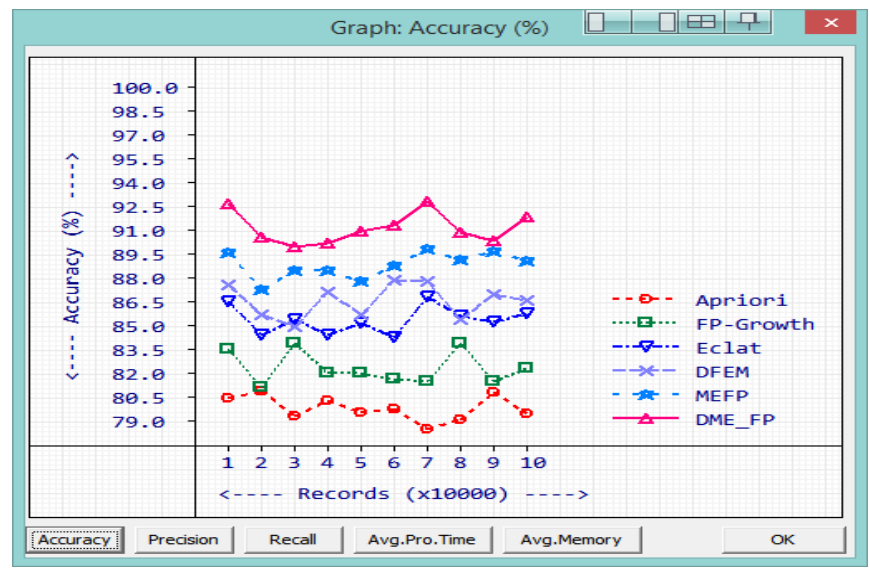

Figure 3

Table 6

\begin{tabular}{||c|c|c|c|c|c|c||}
\hline \multicolumn{7}{|c|}{ Precision $(\%)$} \\
\hline Records & Apriori & FP-Growth & Eclat & DFEM & MEFP & DME-FP \\
\hline 10000 & 80.72 & 83.56 & 87.08 & 87.59 & 89.43 & 92.95 \\
\hline 20000 & 81.13 & 81.52 & 84.23 & 85.63 & 87.34 & 90.73 \\
\hline 30000 & 79.08 & 84.04 & 85.01 & 84.97 & 88.93 & 89.89 \\
\hline 40000 & 80.07 & 82.27 & 84.15 & 87.35 & 88.23 & 90.12 \\
\hline 50000 & 79.33 & 81.94 & 84.9 & 85.51 & 87.47 & 90.76 \\
\hline 60000 & 80.15 & 81.45 & 84.45 & 88.12 & 88.44 & 91.42 \\
\hline 70000 & 78.2 & 81.9 & 86.59 & 88.29 & 89.99 & 93.37 \\
\hline 80000 & 79.55 & 84.19 & 85.5 & 85.81 & 89.13 & 91.44 \\
\hline 90000 & 80.65 & 81.99 & 85.03 & 87.39 & 89.41 & 90.77 \\
\hline 100000 & 79.77 & 82.87 & 85.65 & 87.05 & 88.83 & 91.92 \\
\hline \hline
\end{tabular}

Precision score determines the reliability of a data mining procedure. Higher precision refers higher reliability. DME-FP achieved highest precision value of $93.37 \%$ with precision average of $91.34 \%$ whereas MEFP achieved $89.99 \%$ of highest precision with the average of $88.72 \%$. Precision measurement values for proposed method along with existing methods are given in Table 6 and compared in graph given as Figure 4.

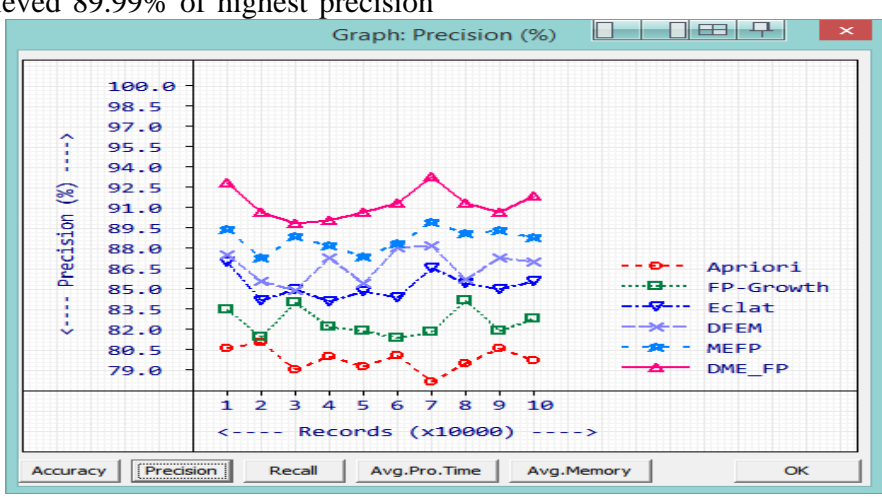

Figure 4

Recall refers the fraction of successfully retrieved relevant items and it is an important parameter in data mining. A good mining procedure should score higher recall values. As per the readings, DME-FP reached $92.8 \%$ recall score with $91.22 \%$ average whereas other existing methods in comparison scored below $90 \%$ of recall value. Observed recall values are given in Table 7 and the comparison chart is given in Figure 5. 
Table 7

\begin{tabular}{||c|c|c|c|c|c|c||}
\hline \hline \multicolumn{7}{|c||}{ Recall (\%) } \\
\hline Records & Apriori & FP-Growth & Eclat & DFEM & MEFP & DME-FP \\
\hline 10000 & 80.46 & 83.45 & 86.13 & 88.13 & 89.8 & 92.8 \\
\hline 20000 & 80.97 & 80.95 & 84.95 & 85.95 & 87.26 & 90.25 \\
\hline 30000 & 79.68 & 83.91 & 85.83 & 85.06 & 88.29 & 90.2 \\
\hline 40000 & 80.6 & 82.55 & 84.5 & 87.45 & 88.4 & 90.37 \\
\hline 50000 & 79.44 & 82.07 & 85.69 & 85.31 & 87.93 & 91.24 \\
\hline 60000 & 79.38 & 81.36 & 84.71 & 88.37 & 89.04 & 91.7 \\
\hline 70000 & 78.24 & 81.88 & 86.52 & 87.84 & 89.48 & 92.8 \\
\hline 80000 & 79.35 & 84.25 & 85.82 & 85.7 & 89.27 & 90.83 \\
\hline 90000 & 80.41 & 81.11 & 84.82 & 86.53 & 89.55 & 90.25 \\
\hline 100000 & 79.4 & 82.88 & 86.36 & 86.83 & 88.99 & 91.79 \\
\hline
\end{tabular}

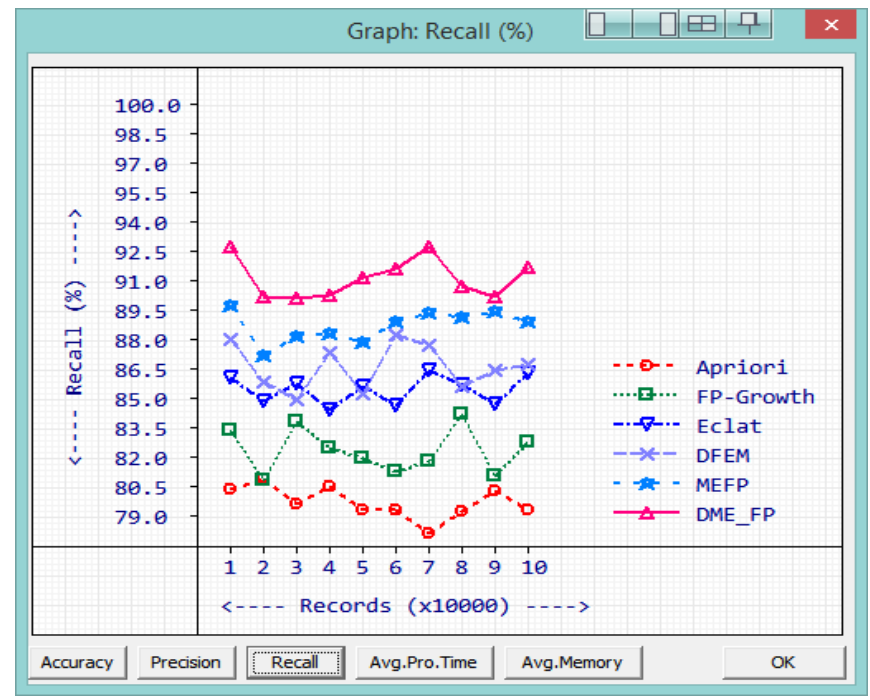

Figure 5

Processing time of a data mining procedure is also a parameter that determines the quality. A good data mining procedure has to produce results with a reasonable time. DME-FP consumed $2918 \mathrm{mS}$ of average processing time to process a data block. Apriori consumes the least time of $2324 \mathrm{mS}$ average processing time which is lesser than DME-FP. FP-Growth, Eclat, DFEM and MEFP consumed $4072 \mathrm{mS}, 3677 \mathrm{mS}, 3548 \mathrm{~ms}$ and $3306 \mathrm{mS}$ respectively. While comparing other methods excluding Apriori, DME-FP consumed lesser time. With the betterment in other parameters the $594 \mathrm{mS}$ time delay can be compromised. Complete processing time readings for all methods are given in Table 8. A comparison graph of processing times is given in Figure 6.

\begin{tabular}{||c|c|c|c|c|c|c||}
\hline \multicolumn{7}{|c|}{ Average Processing Time (mS) } \\
\hline Records & Apriori & FP-Growth & Eclat & DFEM & MEFP & DME-FP \\
\hline 10000 & 2353 & 4096 & 3672 & 3549 & 3400 & 2987 \\
\hline 20000 & 2230 & 4174 & 3618 & 3483 & 3295 & 2870 \\
\hline 30000 & 2338 & 3984 & 3583 & 3575 & 3329 & 2819 \\
\hline 40000 & 2370 & 4049 & 3773 & 3584 & 3251 & 2986 \\
\hline 50000 & 2228 & 4003 & 3610 & 3518 & 3281 & 2991 \\
\hline 60000 & 2308 & 4185 & 3591 & 3509 & 3374 & 3003 \\
\hline 70000 & 2324 & 4147 & 3683 & 3519 & 3210 & 2969 \\
\hline 80000 & 2369 & 4040 & 3756 & 3560 & 3219 & 2826 \\
\hline 90000 & 2414 & 3989 & 3729 & 3557 & 3332 & 2870 \\
\hline 100000 & 2306 & 4053 & 3752 & 3631 & 3366 & 2864 \\
\hline \hline
\end{tabular}


Table 8

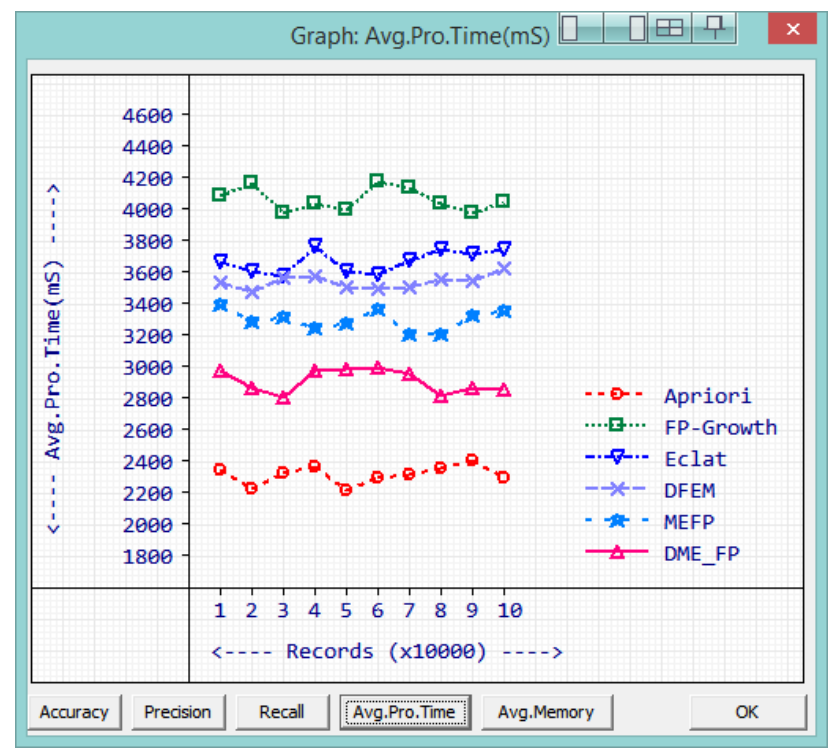

Figure 6

Memory is one of the important computational resources. More memory consumption affects the stability of data mining procedures. While handling large number of item sets and transaction history, consuming more memory will slow down the process. DME-FP consumed 1844 Bytes to process a data block. The nearest performance method MEFP consumed 2304 Bytes. Apriori consumed the least average memory of 1802 Bytes. Average memory consumption to process a data block by all the methods are recorded in table 9. Memory comparison chart is given in Figure 7.

Table 9

\begin{tabular}{||c|c|c|c|c|c|c||}
\hline \multicolumn{7}{|c|}{ Memory (B) } \\
\hline Records & Apriori & FP-Growth & Eclat & DFEM & MEFP & DME-FP \\
\hline 10000 & 1821 & 3586 & 2902 & 2817 & 2312 & 1846 \\
\hline 20000 & 1781 & 3583 & 2905 & 2825 & 2307 & 1846 \\
\hline 30000 & 1787 & 3611 & 2924 & 2848 & 2302 & 1832 \\
\hline 40000 & 1807 & 3614 & 2909 & 2815 & 2302 & 1865 \\
\hline 50000 & 1804 & 3607 & 2930 & 2815 & 2298 & 1821 \\
\hline 60000 & 1792 & 3609 & 2915 & 2832 & 2329 & 1852 \\
\hline 70000 & 1786 & 3598 & 2948 & 2810 & 2320 & 1820 \\
\hline 80000 & 1821 & 3580 & 2927 & 2804 & 2311 & 1858 \\
\hline 90000 & 1809 & 3586 & 2900 & 2826 & 2283 & 1865 \\
\hline 100000 & 1808 & 3610 & 2900 & 2802 & 2284 & 1842 \\
\hline
\end{tabular}




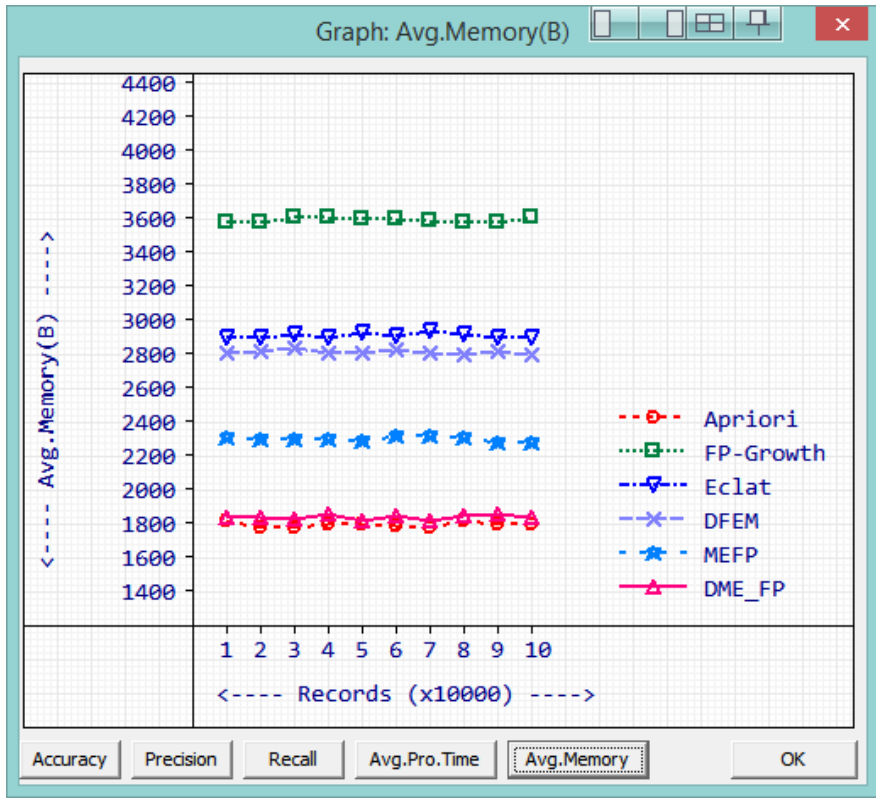

Figure 7

\section{CONCLUSION}

Based on the experimental results DME-FP scored higher in standard data mining parameters of accuracy, precision and recall. The variation in consumption of computational resources like processing time and memory is very lesser to achieve more reliable association rules in proposed DME-FP. The introduction of TPN and RPT results improved performance of proposed DME-FP makes it is more suitable to use in modern data analytical processes.

\section{REFERENCES}

[1] Chien Chiang Lin, Hsing-Hung Lin, Kun-Chih Huang. TRIZ retrospect and prospect. Systems and Informatics (ICSAI) IEEE November 2016

[2] Jeff Heaton. Comparing dataset characteristics that favor the Apriori, Eclat or FP-Growth frequent itemset mining algorithms. IEEE April 2016

[3] Arkan A.G.AL-Hamodi, Songfeng LU, Yahya E.A.ALSalhi. AN ENHANCED FREQUENT PATTERN GROWTH BASED ON MAPREDUCE FOR MINING ASSOCIATION RULES. International Journal of Data Mining \& Knowledge Management Process (IJDKP) March 2016

[4] Dea Delvia Arifin, Shaufiah, Moch.Arif Bijaksana. Enhancing Spam Detection on Mobile Phone Short Message Service (SMS) Performance using FP-Growth and Naive Bayes Classifier. Asia Pacific Conference on Wireless and Mobile (APWiMob). IEEE 2016
[5] Chunkai Zhang, Xudong Zhang, Panbo Tian. An Approximate Approach to Frequent Itemset Mining, Data Science in Cyberspace (DSC). IEEE June 2017

[6] Wan Aezwani Bt Wan Abu Bakar, Zailani B.Abdullah, Md.Yazid B.Md Saman, Masila Bt Abd Jalil, Mustafa B. Man, Tutut Herawan, Abdul Razak Hamdan. IncrementalEclat Model: An Implementation via Benchmark Case Study. Advances in Machine Learning and Signal Processing. Springer June 2016

[7] Iona Sudheendran, Ganesh Kumar R. A Dynamic Approach for Frequent Pattern Mining Using Database Characteristics. International Journal for Research in Applied Science \& Engineering Technology (IJRASET) MAY 2015

[8] Sagar Bhise1, Prof. Sweta Kale, Effieient Algorithms to find Frequent Itemset Using Data Mining, International Research Journal of Engineering and Technology (IRJET) JUNE 2017

[9] Duo Liu, Yi Lin, Po-Chun Huang, Xiao Zhu, Liang Liang. Durable and Energy Efficient In-Memory Frequent Pattern Mining. IEEE Transactions on Computer-Aided Design of Integrated Circuits and Systems, IEEE March 2017

[10] Mukesh Bathre, Vivek Kumar Vaidya, Alok Sahelay. Memory Efficient Frequent Pattern Mining using Transposition of Database. International Journal of Computer Engineering \& Technology (IJCET). APRIL 2016

[11] Data Mining Algorithms In R/Frequent Pattern Mining/The FPGrowthAlgorithm,https://en.wikibooks.org/wiki/Data_M ining_Algorithms_In_R/Frequent_Pattern_Mining/The_FP -Growth_Algorithm

[12] Mahito Sugiyama, Karsten M. Borgwardt, Significant Pattern Mining on Continuous Variables. Cornell University Library 2017

[13] Ian H. Witten, Eibe Frank, Mark A. Hall, Christopher J. Pal. Data Mining: Practical machine learning tools and techniques. Fourth Edition

[14] Mahsa Salehi, Christopher Leckie, James C.Bezdek, Tharshan Vaithianathan, Xuyun Zhang. Fast Memory Efficient Local Outlier Detection in Data Streams, IEEE Transactions on Knowledge and Data Engineering, IEEE December 2016

[15] Souleymane Zida, Philippe Fournier-Viger, Jerry ChunWei Lin, Cheng-Wei Wu, Vincent S. Tseng. EFIM: a fast and memory efficient algorithm for high-utility itemset mining. Knowledge and Information Systems. Springer May,2017 